\title{
Instability of an interface between air and a low conducting liquid subjected to charge injection
}

\author{
Rafael Chicón \\ Departamento de Física, Universidad de Murcia, Apdo. 4021, 30071 Murcia, Spain
}

Alberto T. Pérez

Dpto. Electrónica y Electromagnetismo, Facultad de Física, Reina Mercedes s/n, 41012 Sevilla, Spain

(Received 7 March 2006; accepted 7 September 2006; published online 24 October 2006)

\begin{abstract}
We study the linear stability of an interface between air and a low conducting liquid in the presence of unipolar injection of charge. As a consequence of charge injection, a volume charge density builds up in the air gap and a surface charge density on the interface. Above a certain voltage threshold the electrical stresses may destabilize the interface, giving rise to a characteristic cell pattern known as rose-window instability. Contrary to what occurs in the classical volume electrohydrodynamic instability in insulating liquids, the typical cell size is several times larger than the liquid depth. We analyze the linear stability through the usual procedure of decomposing an arbitrary perturbation into normal modes. The resulting homogeneous linear system of ordinary differential equations is solved using a commercial software package. Finally, an analytical method is developed that provides a solution valid in the limit of small wavenumbers. (C) 2006 American Institute of Physics. [DOI: 10.1063/1.2363219]
\end{abstract}

\section{INTRODUCTION}

When a layer of low conducting liquid is subjected to a corona discharge in air, for example, in a point-to-plate geometry, there appear instabilities of the liquid surface. ${ }^{1-4}$ These instabilities are clearly associated to the electric forces that act upon the liquid. If the liquid conductivity is very low, a space charge distribution is developed in the bulk and the forces are volume forces. This is the case when the electric relaxation time in the liquid $\epsilon / \sigma$ is greater than the time of transit of ions through the liquid layer thickness, $d /\left(K_{l} E\right)$ ( $\epsilon$ is the liquid permittivity, $\sigma$ the liquid conductivity, $K_{l}$ the ion mobility in the liquid, $d$ the liquid layer thickness, and $E$ the average electric field in the liquid). Under these circumstances, an ion can traverse the liquid layer before being neutralized, there is net electric charge in the bulk and the electric conduction regime is not Ohmic. Taking as typical values $K_{l}=10^{-9} \mathrm{~m}^{2} / \mathrm{V} \mathrm{s}, \epsilon=10^{-11} \mathrm{~F} / \mathrm{m}, d=10^{-3} \mathrm{~m}$, and $V=100 \mathrm{~V}$, a conductivity of less than $10^{-12} \mathrm{~S} / \mathrm{m}$ is needed in order to fulfill this condition (it should be noted that this value depends on the voltage, as well as on the geometrical configuration). The kind of instability that appears in such a good insulating liquid layer is referred to as the instability induced by unipolar injection. This is a classical electrohydrodynamic (EHD) instability, and it has been the subject of many experimental as well as theoretical studies. ${ }^{5-7}$ It is characterized by an hexagonal pattern of cells with a typical size of the order of the liquid layer thickness, and the liquid motion starts at a definite value of the potential drop through the liquid. Several devices can be envisaged to inject charge in the liquid. In the experiments where the injection is achieved by corona discharge, the voltage drop through the liquid is determined by the corona current. ${ }^{3}$

On the other hand, if the electric relaxation time is smaller than the time of transit of ions, any charge present in the volume is neutralized before it can travel a significant distance. The liquid volume is electrically neutral and the conduction regime is Ohmic. Now, the classical EHD instability due to unipolar injection is not possible, since there is no electrical volume force. However, there is surface charge on the liquid-air interface and the electric field acts upon this charge via the electrical stresses. Dealing with liquids in the Ohmic regime, but still low conductivity, the surface charge is of the same sign as the corona current. The electric field pushes the liquid against the plate and can disturb the surface. Experimental observations ${ }^{1-3}$ show that an EHD instability emerges that is characterized by a pattern of cells with a typical size several times the liquid layer thickness. This instability has been observed in a point-to-plate geometry, and the non-homogeneous field acting onto the liquid surface produces a variable cell size: the cells are smaller at the center and their size increases outward. This variable size precludes the formation of a perfect hexagonal pattern, and the whole liquid surface takes an appearance that reminds that of a rose window in a Gothic cathedral. This is why this instability is referred to as the rose-window instability. ${ }^{2}$

Besides the size of the cells, other characteristics distinguish experimentally the rose-window instability from the EHD volume instability. They are associated more to the value of the electric field at the liquid interface than to the corona current. Also, the size of the cell neatly decreases when the electric field increases, whereas in the EHD volume instability this size remains largely unchanged.

The point-to-plate geometry is a difficult one for a theoretical analysis. Another complication from the theoretical point of view is associated with the existence of the corona threshold, below which there is no corona current. Fortunately, some experiments have been carried out in a triode configuration: point-grid plate. ${ }^{3}$ The voltage applied to the 
grid helps to control the corona current, and allows us to have a corona current for any value of the grid-to-plate voltage. Moreover, the grid-plate configuration can be reasonably approximated by two infinite parallel planes. This is the configuration studied in this work, where two infinite horizontal electrodes are considered. The upper electrode is the injecting one and is in contact with the air. This electrode simulates the grid, and the injection process the corona current that passes through the grid. The liquid is disposed onto the lower electrode. We address the problem of the electrohydrodynamic stability of the liquid layer, when a voltage difference is applied between both electrodes, and there is charge injection from the upper one.

Interfacial electrohydrodynamic instabilities have been the object of classical studies in electrohydrodynamics, like those of Taylor and McEwan ${ }^{8}$ or Melcher and Smith, ${ }^{9}$ and they are reviewed in the book by Melcher. ${ }^{10}$ However, in all these works the conduction regime is considered to be Ohmic. Therefore, the ratio of conductivities or permittivities determined the surface charge and the electrical stresses. The problem considered in this paper differs from that studied by Melcher ${ }^{9}$ in the appearance of surface charge due to the injection from the upper medium. This injection process introduces another parameter and makes the problem more complex. We will try, when possible, to compare our results with the corresponding limit situations in the classical EHD studies.

A theoretical study of the instability of a non-Ohmic/ Ohmic fluid interface has already been published. ${ }^{11}$ The basic equations we write now are the same as set in that work. However, in Ref. 11 the liquid motion was neglected under the hypothesis that the balance of normal stresses determines the instability threshold, the tangential stresses being negligible. But shear stresses are strictly negligible only under certain conditions, and their role should be analyzed. ${ }^{12} \mathrm{We}$ now take into account the liquid motion in the stability analysis. We will show that, although some of the main conclusions in Ref. 11 still hold, the critical values are noticeably affected by the inclusion of the velocity in the calculations.

In Sec. II we write the basic equations, obtain the unperturbed solution, and introduce linear perturbations and normal mode analysis. Section III presents the numerical method employed to solve the equations and the results obtained. In Sec. IV we develop an analytical method valid in the limit of a small wavenumber. The most significant limit situations are also studied in this section. Finally in Sec. V we recall the main conclusions of the paper.

\section{POSITION OF THE PROBLEM}

\section{A. Basic equations}

We consider two infinite horizontal parallel plane electrodes a distance $h$ apart, and subjected to a potential difference $V$. Over the lower electrode there is disposed a liquid layer of thickness $d$ and finite conductivity $\sigma$. Air fills the upper part of the gap and charge is injected from the upper electrode. Other liquid properties are denoted as $\rho$ for density, $\eta$ for viscosity, $\gamma$ for surface tension, and $\epsilon$ for the dielectric constant. We choose our coordinates in such a way that the vertical axis is $z, z=0$ corresponds to the unperturbed liquid surface, $z=d$ to the lower electrode, and $z=-L(h=d$ $+L)$ to the upper one.

The electrical equations in the airgap are

$$
\begin{aligned}
& \nabla^{2} \phi=-\frac{q}{\epsilon_{0}}, \\
& \frac{\partial q}{\partial t}+\nabla \cdot \mathbf{J}=0 .
\end{aligned}
$$

Here $\mathbf{J}=K q \mathbf{E}, q$ stands for charge density, $K$ for ion mobility, $\mathbf{E}$ for the electric field, and $\phi$ for the electric potential. The transport of charge in air is non-Ohmic and a spatial charge density is built. An average mobility $K$ is assumed for the ions. The drift velocity of ions in air is usually much greater than the fluid velocity, and this is why we neglect the convection term $(q \mathbf{u})$ in Eq. (2). Also, charge diffusion is neglected (see Ref. 7 for details).

The mechanical equation in the air is assumed to be that of the hydrostatic equilibrium:

$$
-\nabla p+\rho_{a} g \mathbf{e}_{z}+q \mathbf{E}=0,
$$

where $p$ is the pressure, $\rho_{a}$ the air mass density, $g$ the acceleration of gravity, and $\mathbf{e}_{z}$ the unit vector in the $z$ direction. Once the liquid surface is perturbed, this equation cannot be but an approximation. In effect, any wavy perturbation of the liquid surface will produce a gradient of the charge density parallel to the electrodes. In that case the last term of the left-hand side of the equation will have a nonzero curl, $\nabla q \times \mathbf{E}$. Therefore, it cannot be balanced by a pressure term. However, air movement above the liquid surface has a small influence on the liquid, due to the difference in viscosity of both fluids, and we can go ahead, as a first approximation, without computing the velocity field in air.

The electrical equations for the liquid are

$$
\nabla^{2} \phi=0
$$

$\mathbf{J}=\sigma \mathbf{E}$,

and the mechanical equations:

$\boldsymbol{\nabla} \cdot \mathbf{u}=0$

$$
\rho\left(\frac{\partial \mathbf{u}}{\partial t}+\mathbf{u} \cdot \nabla \mathbf{u}\right)=-\nabla p+\rho g \mathbf{e}_{z}+\eta \nabla^{2} \mathbf{u} .
$$

To these equations we have to add suitable boundary conditions. Assuming that the electrodes are rigid and perfect conductors, the boundary conditions at both electrodes are as follows: 


$$
\begin{aligned}
& \phi=V, \quad \text { at } \quad z=-L, \\
& q=q_{0}, \quad \text { at } \quad z=-L, \\
& \phi=0, \quad \text { at } \quad z=d, \\
& \mathbf{u}=0, \quad \text { at } \quad z=d .
\end{aligned}
$$

The condition on the charge density at the injecting electrode, where we assume that $q_{0}$ does not depend on the electric field, is referred to as the autonomous injection hypothesis. When the injected charge density is large enough all the electrical quantities become independent of the level of injection, the so-called strong injection limit, and that hypothesis is no longer relevant. This limit is often encountered in the experiments.

At the interface we have the following boundary conditions (the brackets $[A]=A_{l}-A_{a}$ denote the jump of a quantity $A$ across the interface):

$$
\begin{aligned}
& {[\mathbf{E}] \times \mathbf{n}=0,} \\
& {[\epsilon \mathbf{E}] \cdot \mathbf{n}=q_{s},}
\end{aligned}
$$

where $\epsilon$ is the electrical permittivity of the corresponding medium, $q_{s}$ is the surface charge density, and $\mathbf{n}$ the unit vector normal to the surface and pointing toward the liquid. The charge conservation equation at the interface is

$$
\frac{\partial q_{s}}{\partial t}+\nabla_{s} \cdot\left(q_{s} \mathbf{u}\right)+[\mathbf{J}] \cdot \mathbf{n}-[q \mathbf{u}] \cdot \mathbf{n}=0,
$$

where $\boldsymbol{\nabla}_{s}$ is the divergence operator restricted to the surface. ${ }^{12}$ An alternative, but equivalent, form of this conservation equation may be found in. ${ }^{13}$ The kinematic condition, expressing that the surface is a material one, is

$$
-\frac{\partial f}{\partial t}+u_{z}+\mathbf{u} \cdot \nabla_{s} f=0
$$

where the interface is defined by the function $z=f(x, y)$.

Finally, we have to impose the balance of tangential and normal total stresses, that is,

$$
\eta \mathbf{t} \cdot\left(\boldsymbol{\nabla} \mathbf{u}+\nabla \mathbf{u}^{T}\right) \cdot \mathbf{n}+q_{s} \mathbf{E} \cdot \mathbf{t}=0,
$$

where $\mathbf{t}$ is a unit vector tangent to the interface, and

$$
\begin{aligned}
-[p] & +\eta \mathbf{n} \cdot\left(\boldsymbol{\nabla} \mathbf{u}+\boldsymbol{\nabla} \mathbf{u}^{T}\right) \cdot \mathbf{n}+\left[\epsilon(\mathbf{E} \cdot \mathbf{n})^{2}\right]-\left[\frac{1}{2} \epsilon E^{2}\right] \\
& -\gamma\left(\nabla_{s} \cdot \mathbf{n}\right)=0 .
\end{aligned}
$$

These two conditions play a key role in the problem. Some essential features of the rose-window instability are consequences of the balance of normal stresses. On the other hand, as soon as the liquid surface is disturbed there appear electric tangential stresses, which can only be compensated by the liquid motion. Only in certain limit circumstances, for example in the case of a perfect conductor, is it safe to neglect the liquid velocity.

\section{B. Nondimensional equations}

We take as units $d$ for distances, $\rho d^{2} / \eta$ for time, $\eta^{2} /\left(\rho d^{2}\right)$ for pressure, $V / d$ for the electric field, and $\epsilon_{0} V / d^{2}$ for charge. With this choice of units, and denoting the nondimensional quantities with the same symbols as the dimensional ones, the nondimensional equations in the air are

$$
\begin{aligned}
& \nabla^{2} \phi=-q, \\
& \frac{M}{U^{1 / 2}} \frac{\partial q}{\partial t}+\nabla \cdot(q \mathbf{E})=0, \\
& -\nabla p+\frac{\rho_{a}}{\rho} g^{*} \mathbf{e}_{z}+U q \mathbf{E}=0,
\end{aligned}
$$

and in the liquid we have

$$
\begin{aligned}
& \nabla^{2} \phi=0, \\
& \mathbf{J}=S \mathbf{E}, \\
& \frac{\partial \mathbf{u}}{\partial t}+\mathbf{u} \cdot \nabla \mathbf{u}=-\nabla p+g^{*} \mathbf{e}_{z}+\nabla^{2} \mathbf{u} .
\end{aligned}
$$

The boundary conditions at the electrodes are

$$
\begin{aligned}
& \phi=1, \quad \text { at } \quad z=-L, \\
& q=C, \quad \text { at } \quad z=-L, \\
& \phi=0, \quad \text { at } \quad z=1, \\
& \mathbf{u}=0, \quad \text { at } \quad z=1,
\end{aligned}
$$

and at the interface,

$$
[\mathbf{E}] \times \mathbf{n}=0,
$$

$$
\begin{aligned}
& {\left[\frac{\boldsymbol{\epsilon}}{\epsilon_{0}} \mathbf{E}\right] \cdot \mathbf{n}=q_{s},} \\
& \frac{M}{U^{1 / 2}}\left(\frac{\partial q_{s}}{\partial t}+\nabla_{s} \cdot\left(q_{s} \mathbf{u}\right)-[q \mathbf{u}] \cdot \mathbf{n}\right)+[\mathbf{J}] \cdot \mathbf{n}=0,
\end{aligned}
$$

$$
\begin{aligned}
& -\frac{\partial f}{\partial t}+u_{z}+\nabla_{s} f \cdot \mathbf{u}=0, \\
& \mathbf{t} \cdot\left(\boldsymbol{\nabla} \mathbf{u}+\boldsymbol{\nabla} \mathbf{u}^{T}\right) \cdot \mathbf{n}+U q_{s} \mathbf{E} \cdot \mathbf{t}=0, \\
& -[p]+\mathbf{n} \cdot\left(\boldsymbol{\nabla u}+\nabla \mathbf{u}^{T}\right) \cdot \mathbf{n}+U\left(\left[\frac{\epsilon}{\epsilon_{0}}(\mathbf{E} \cdot \mathbf{n})^{2}\right]\right. \\
& \left.\quad-\left[\frac{1}{2} \frac{\epsilon}{\epsilon_{0}} E^{2}\right]\right)-\frac{g^{*}}{\mathrm{Bo}}\left(\nabla_{s} \cdot \mathbf{n}\right)=0 .
\end{aligned}
$$

The following nondimensional parameters govern the problem: 


$$
\begin{aligned}
& U=\frac{\epsilon_{0} \rho V^{2}}{\eta^{2}}, \quad M=\frac{1}{K} \sqrt{\frac{\epsilon_{0}}{\rho}}, \quad C=\frac{q_{0} d^{2}}{\epsilon_{0} V}, \\
& S=\frac{\sigma d^{2}}{K \epsilon_{0} V}, \quad g^{*}=\frac{\rho^{2} d^{3}}{\eta^{2}} g, \quad \text { Во }=\frac{\rho g d^{2}}{\gamma} .
\end{aligned}
$$

The parameter $U$ is the ratio of the electric to the viscous forces in the liquid. Parameter $M^{-1}$ is a nondimensional measure of the mobility of the ions in air. Parameter $C$ measures the level of injection, for it compares the injected charge per unit area $q_{0} d$ with the surface charge density $\epsilon_{0} V / d$ induced in the electrodes in the absence of injection. Parameter $S$ is a nondimensional group proportional to the liquid conductivity. If air were treated as an Ohmic conductor with some definite conductivity, $S$ would simply be the ratio between both conductivities. But air is not an Ohmic conductor, hence the relative importance of the liquid conductivity depends on the applied voltage. Finally, $g^{*}$ is a nondimensional measure of the acceleration of gravity, $\epsilon_{r}$ is the relative permittivity of the liquid, and Bo is the usual Bond number representing the ratio between the gravity and capillary forces.

\section{Static solution}

Equations (15)-(20) admit a static solution, which is the starting point for the stability analysis. This solution corresponds to zero liquid velocity, a flat liquid interface, and a steady current through both fluids, air and liquid. The solutions for the electric field and charge density are, in the air, as follows:

$$
\begin{aligned}
& E_{0 z}(z)=a(z+b)^{1 / 2}, \\
& Q_{0}(z)=\frac{a / 2}{(z+b)^{1 / 2}},
\end{aligned}
$$

and in the liquid,

$$
\begin{aligned}
& E_{0 z}=\frac{a^{2}}{2 S}, \\
& Q_{0}=0 .
\end{aligned}
$$

The electric current through the system and the surface charge at the interface are

$$
\begin{aligned}
& J_{0}=\frac{a^{2}}{2}, \\
& Q_{s 0}=\epsilon_{r} \frac{a^{2}}{2 S}-a b^{1 / 2} .
\end{aligned}
$$

The pressure is, in the air,

$$
p_{0}(z)=P_{0 a}+\left(\frac{\rho_{a}}{\rho} g^{*}+U J_{0}\right)(z+L),
$$

and in the liquid,

$$
p_{0}(z)=P_{0 l}+g^{*} z,
$$

$P_{0 a}$ and $P_{0 l}$ being constants. The constants $a$ and $b$ are functions of the parameters $S, L$, and $C$, and are implicitly given by the equations

$$
\begin{aligned}
& \frac{a^{2}}{2 S}+\frac{2}{3} a\left[b^{3 / 2}-(b-L)^{3 / 2}\right]=1, \\
& \frac{a / 2}{(b-L)^{1 / 2}}=C .
\end{aligned}
$$

Equation (34) shows that for low values of $S$ the charge density is positive, i.e., of the same sign as the injector. Hence the electric field pushes the liquid against the lower electrode. For large values of $S$ the situation is reversed, and the charge is of opposite sign to that of the injector. In that case the field pulls the liquid up. This is the usual situation when dealing with good conductors. There is a definite value of $S$ for which the charge density is zero.

It is interesting to compute the electric pressure jump at the interface:

$$
\frac{1}{2} \epsilon_{r} E_{0 z}^{2}(0+)-\frac{1}{2} E_{0 z}^{2}(0-)=\frac{1}{2} a^{2}\left(\epsilon_{r} \frac{a^{2}}{4 S^{2}}-b\right) .
$$

As expected, it also changes sign with $S$, although for a different value from that corresponding to zero surface charge. The equations that implicitly define both transition values differ by a factor $\sqrt{\epsilon_{r}}$. It can be shown that, for small $S$, the electric pressure jump is a decreasing function of the liquid layer thickness. This is a potentially unstable situation, because if we perturb the surface, those regions where the liquid is thinner will suffer a greater pressure. Something similar, but with the sign of the pressure jump reversed, occurs for the case of large values of $S$.

\section{Linearization}

To carry out a linear stability analysis of the unperturbed state, we introduce the following perturbation variables:

$$
\begin{aligned}
& \phi(x, y, z, t)=\Phi_{0}(z)+\phi^{\prime}(x, y, z, t), \\
& \mathbf{E}(x, y, z, t)=\mathbf{E}_{0}(z)+\mathbf{E}^{\prime}(x, y, z, t), \\
& q(x, y, z, t)=Q_{0}(z)+q^{\prime}(x, y, z, t), \\
& p(x, y, z, t)=p_{0}(z)+p^{\prime}(x, y, z, t), \\
& J=J_{0}+J^{\prime} .
\end{aligned}
$$

The introduction of these variables in Eqs. (15)-(20) yields, upon linearization, in the air,

$$
\nabla^{2} \phi^{\prime}=-q^{\prime},
$$

$$
\begin{aligned}
& \frac{M}{U^{1 / 2}} \frac{\partial q^{\prime}}{\partial t}+\frac{d Q_{0}}{d z} E_{z}^{\prime}+2 Q_{0} q^{\prime}+E_{0} \frac{\partial q^{\prime}}{\partial z}=0, \\
& -\nabla p^{\prime}+U\left(Q_{0} \mathbf{E}^{\prime}+q^{\prime} \mathbf{E}_{0}\right)=0,
\end{aligned}
$$

and in the liquid, 


$$
\begin{aligned}
& \nabla^{2} \phi^{\prime}=0, \\
& \mathbf{J}^{\prime}=S \mathbf{E}^{\prime}, \\
& \frac{\partial \mathbf{u}}{\partial t}=-\nabla p^{\prime}+\nabla^{2} \mathbf{u} .
\end{aligned}
$$

The pressure term in Eq. (46) can be eliminated taking twice the curl in both sides, giving

$$
\left(\nabla^{2}-\frac{\partial}{\partial t}\right) \nabla^{2} \mathbf{u}=0
$$

In order to linearize the boundary conditions at the interface, we follow the usual procedure of extending the functions outside its initial domain by means of a Taylor expansion. $^{14}$ For example, the electric potential at $z_{s}=f(x, y, t)$ is

$$
\phi\left(x, y, z_{s}, t\right)=\phi(x, y, 0, t)+\left(\frac{\partial \phi}{\partial z}\right)_{(z=0)} f(x, y, t)+\ldots .
$$

Introducing the perturbation and neglecting second order terms,

$$
\begin{aligned}
\phi\left(x, y, z_{s}, t\right)= & \Phi_{0}(x, y, 0, t)+\phi^{\prime}(x, y, 0, t) \\
& +\left(\frac{\partial \Phi_{0}}{\partial z}\right)_{(z=0)} f(x, y, t)+\ldots .
\end{aligned}
$$

We follow the same procedure for any quantity defined at the interface. The boundary conditions become

$$
\begin{aligned}
& {\left[\phi^{\prime}\right]_{0}=-\left[\frac{d \Phi_{0}}{d z}\right]_{0},} \\
& {\left[\frac{\epsilon}{\epsilon_{0}} \frac{\partial \phi^{\prime}}{\partial z}\right]_{0}=-q_{s}^{\prime}-\left[\frac{\epsilon}{\epsilon_{0}} \frac{d^{2} \Phi_{0}}{d z^{2}}\right]_{0},} \\
& \frac{M}{U^{1 / 2}}\left(\frac{\partial q_{s}^{\prime}}{\partial t}-Q_{s 0} \frac{\partial u_{z}}{\partial z}(0+)\right)+S E_{z}^{\prime}(0+) \\
& -Q_{0}(0-) E_{z}^{\prime}(0-)-E_{0 z}(0-) q^{\prime}(0-)=0, \\
& -\frac{\partial f}{\partial t}+u_{z}(0+)=0, \\
& \nabla_{s}^{2} u_{z}(0+)-\frac{\partial^{2} u_{z}}{\partial z^{2}}(0+) \\
& -U Q_{s 0}\left(\nabla_{s}^{2} \phi^{\prime}(0+)+\frac{d \Phi_{0}}{d z}(0+) \nabla_{s}^{2} f\right)=0, \\
& \left.-\left[p^{\prime}\right]_{0}-\left(1-\frac{\rho_{a}}{\rho}\right) g E_{0 z}^{*} \frac{\partial \phi^{\prime}}{\partial z}\right]_{0}=0, \frac{\partial u_{z}}{\partial z}(0+)+\frac{g^{*}}{\mathrm{Bo}} \nabla_{s}^{2} f
\end{aligned}
$$

Eq. (49) allows us to eliminate $q_{s}^{\prime}$ in Eq. (50), which becomes

$$
\begin{aligned}
& \frac{M}{U^{1 / 2}}\left(-Q_{s 0} \frac{\partial u_{z}}{\partial z}(0+)-\left[\frac{\epsilon}{\epsilon_{0}} \frac{\partial}{\partial t} \frac{\partial \phi^{\prime}}{\partial z}\right]_{0}-\left[\frac{\epsilon}{\epsilon_{0}} \frac{d^{2} \Phi_{0}}{d z^{2}}\right]_{0} \frac{\partial f}{\partial t}\right) \\
& \quad+S E_{z}^{\prime}(0+)-Q_{0}(0-) E_{z}^{\prime}(0-)-E_{0 z}(0-) q^{\prime}(0-) \\
& \quad=0 .
\end{aligned}
$$

Some algebraic steps allow us also to eliminate the pressure $p^{\prime}$ in Eq. (53). Taking the $x$ derivative of the $x$ component of Eq. (46), the $y$ derivative of the $y$ component and adding the results one obtains

$$
-\nabla_{s}^{2} p^{\prime}=\frac{\partial^{3} u_{z}}{\partial z^{3}}+\nabla_{s}^{2} \frac{\partial u_{z}}{\partial z}-\frac{\partial}{\partial t} \frac{\partial u_{z}}{\partial z}
$$

where the continuity equation has been used to eliminate $u_{x}$ and $u_{y}$. Following a similar procedure with Eq. (43) in the air yields

$$
-\nabla_{s}^{2} p^{\prime}=U Q_{0} \nabla_{s}^{2} \phi^{\prime}
$$

These expressions permit us to obtain the jump at the interface $\left[\nabla_{s}^{2} p^{\prime}\right]_{0}$. Applying the operator $\nabla_{s}^{2}$ to Eq. (53) and substituting for $\left[\nabla_{s}^{2} p^{\prime}\right]_{0}$, one finally obtains

$$
\begin{aligned}
\left(3 \nabla_{s}^{2}-\frac{\partial}{\partial t}\right) \frac{\partial u_{z}}{\partial z}(0+)+\frac{\partial^{3} u_{z}}{\partial z^{3}}(0+) & \\
& -U\left(Q_{0}(0-) \nabla_{s}^{2} \phi^{\prime}(0-)+\left[\frac{\epsilon}{\epsilon_{0}} E_{0} \nabla_{s}^{2} \frac{\partial \phi^{\prime}}{\partial z}\right]_{0}\right) \\
& -\left(1-\frac{\rho_{a}}{\rho}\right) g^{\star} \nabla_{s}^{2} f+\frac{g^{\star}}{\mathrm{Bo}}\left(\nabla_{s}^{2}\right)^{2} f=0 .
\end{aligned}
$$

\section{E. Normal mode analysis}

The above equations are linear and their coefficients do not depend on $x, y$, or $t$. Therefore, they are amenable to a normal-mode analysis. Accordingly, we assume the perturbation variables to have the form

$$
\begin{aligned}
& f(x, y, t)=f_{0} e^{\omega t} e^{i\left(k_{x} x+k_{y} y\right)}, \\
& \phi^{\prime}(x, y, z, t)=g(z) e^{\omega t} e^{i\left(k_{x} x+k_{y} y\right)}, \\
& u_{z}(x, y, z, t)=u(z) e^{\omega t} e^{i\left(k_{x} x+k_{y} y\right)},
\end{aligned}
$$

and obtain the following set of equations, in the air:

$$
\left(\frac{M}{U^{1 / 2}} \omega+2 Q_{0}(z)+E_{0}(z) \frac{d}{d z}\right)\left(\frac{d^{2}}{d z^{2}}-k^{2}\right) g+\frac{d Q_{0}}{d z} \frac{d g}{d z}=0,
$$

in the liquid:

$$
\begin{aligned}
& \left(\frac{d^{2}}{d z^{2}}-k^{2}\right) g=0 \\
& \left(\frac{d^{2}}{d z^{2}}-k^{2}-\omega\right)\left(\frac{d^{2}}{d z^{2}}-k^{2}\right) u=0,
\end{aligned}
$$

and for the interface:

$$
[g]_{0}=\left[E_{0}\right]_{0} f_{0},
$$




$$
\begin{aligned}
& -\frac{M}{U^{1 / 2}}\left(Q_{s 0} \frac{d u}{d z}(0+)+\omega\left[\frac{\epsilon}{\epsilon_{0}} \frac{d g}{d z}\right]_{0}+\omega f_{0}\left[\frac{\epsilon}{\epsilon_{0}} \frac{d^{2} \Phi_{0}}{d z^{2}}\right]_{0}\right) \\
& -S \frac{d g}{d z}(0+)+Q_{0}(0-) \frac{d g}{d z}(0-)+E_{0}(0-) \\
& \quad \times\left(\frac{d^{2} g}{d z^{2}}-k^{2} g\right)(0-)=0 \\
& -\omega f_{0}+u(0+)=0 \\
& \frac{d^{2} u}{d z^{2}}(0+)+k^{2}\left\{u(0+)+U Q_{s 0}\left[E_{0}(0+) f_{0}-g(0+)\right]\right\}=0,
\end{aligned}
$$

$$
\begin{aligned}
& \frac{d^{3} u}{d z^{3}}(0+)-\left(3 k^{2}+\omega\right) \frac{d u}{d z}(0+) \\
& \quad+U k^{2}\left(Q_{0}(0-) g(0-)+\left[\frac{\epsilon}{\epsilon_{0}} E_{0} \frac{d g}{d z}\right]\right) \\
& \quad+k^{2}\left(1-\frac{\rho_{a}}{\rho}+\frac{k^{2}}{\mathrm{Bo}}\right) g^{*} f_{0}=0 .
\end{aligned}
$$

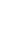

We assume that the principle of exchange of stabilities holds in this problem, so that marginal stability occurs for $\omega=0 .{ }^{15}$ Throughout the rest of the work we shall be concerned with the study of only marginally stable states.

Up to here our equations were defined on the interval $(-L, 0)$ for the air and $(0,1)$ for the liquid. It is convenient to have all functions defined on the same interval. Therefore, we define $h(z)=g(-L z)$ and finally get the following set of equations on the domain $0<z<1$ :

$$
\begin{aligned}
& {\left[\frac{d^{3}}{d z^{3}}-\frac{L}{b-L z} \frac{d^{2}}{d z^{2}}-L^{2}\left(k^{2}+\frac{1}{4} \frac{1}{(b-z L)^{2}}\right) \frac{d}{d z}\right.} \\
& \left.\quad+L^{3} \frac{k^{2}}{b-L z}\right] h(z)=0, \\
& \left(\frac{d^{2}}{d z^{2}}-k^{2}\right) g(z)=0, \\
& \left(\frac{d^{2}}{d z^{2}}-k^{2}\right)^{2} u(z)=0,
\end{aligned}
$$

with the following boundary conditions:

$$
\begin{aligned}
& h(1)=0, \\
& \frac{d^{2} h}{d z^{2}}(1)=0, \\
& g(1)=0, \\
& u(1)=0, \\
& \frac{d u}{d z}(1)=0,
\end{aligned}
$$

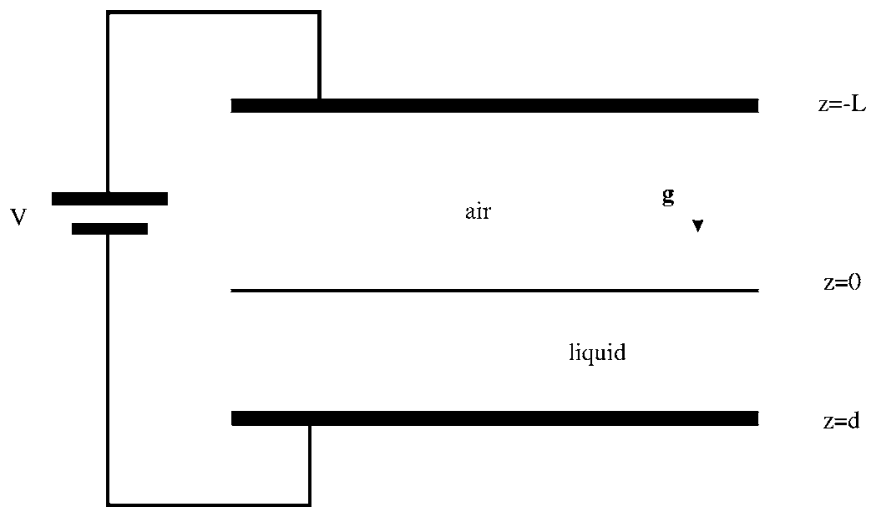

FIG. 1. Geometrical configuration of the system.

$$
\begin{aligned}
& g(0)-h(0)=\left(\frac{a^{2}}{2 S}-a b^{1 / 2}\right) f_{0}, \\
& \frac{M}{U^{1 / 2}}\left(\epsilon_{r} \frac{a^{2}}{2 S}-a b^{1 / 2}\right) \frac{d u}{d z}(0)+S \frac{d g}{d z}(0)-\frac{a b^{1 / 2}}{L^{2}} \frac{d^{2} h}{d z^{2}}(0) \\
& +\frac{a}{2 L b^{1 / 2}} \frac{d h}{d z}(0)+k^{2} a b^{1 / 2} h(0)=0, \\
& u(0)=0, \\
& \frac{d^{2} u}{d z^{2}}(0)+k^{2} U\left(\epsilon_{r} \frac{a^{2}}{2 S}-a b^{1 / 2}\right)\left(\frac{a^{2}}{2 S} f_{0}-g(0)\right)=0, \\
& \frac{d^{3} u}{d z^{3}}(0)-3 k^{2} \frac{d u}{d z}(0)+k^{2} U\left(\epsilon_{r} \frac{a^{2}}{2 S} \frac{d g}{d z}(0)+\frac{a b^{1 / 2}}{L} \frac{d h}{d z}(0)\right. \\
& \left.+\frac{a}{2 b^{1 / 2}} h(0)\right)+k^{2}\left(1-\frac{\rho_{a}}{\rho}+\frac{k^{2}}{\mathrm{Bo}}\right) g^{*} f_{0}=0 .
\end{aligned}
$$

Thus we have to solve a ninth order homogeneous system of ODE's with ten boundary conditions.

\section{NUMERICAL SOLUTION AND RESULTS}

The boundary value problem formulated above is approached by utilizing a boundary value problem solver available in version 6.1 of MATLAB, namely bvp4c. ${ }^{16}$ This MATLAB function implements a collocation method that uses cubic splines to approximate the solution.

The algorithm is particularly well suited for the computation of the dispersion relation $U(k)$, due to the possibility of taking advantage of a continuation technique. The method consists in using the solution obtained for a given value of $k$ as an initial guess for the solution corresponding to the next value of $k$. The continuation technique is also most useful when checking the robustness of the solution. The solution computed with a given error tolerance (a parameter that is chosen by the user) is used as the initial guess for a new computation with a more stringent error tolerance. If the results keep stable, we can be confident about the solution thus obtained.

Typical dispersion diagrams $U(k)$ are shown in Figs. 2(a) and 2(b), corresponding to values of the parameters for 


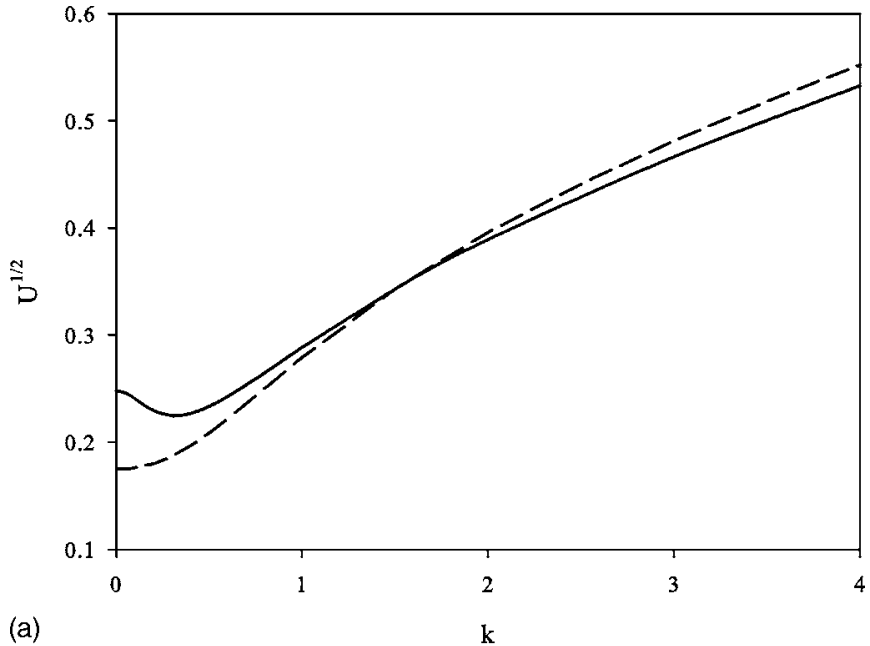

(a)

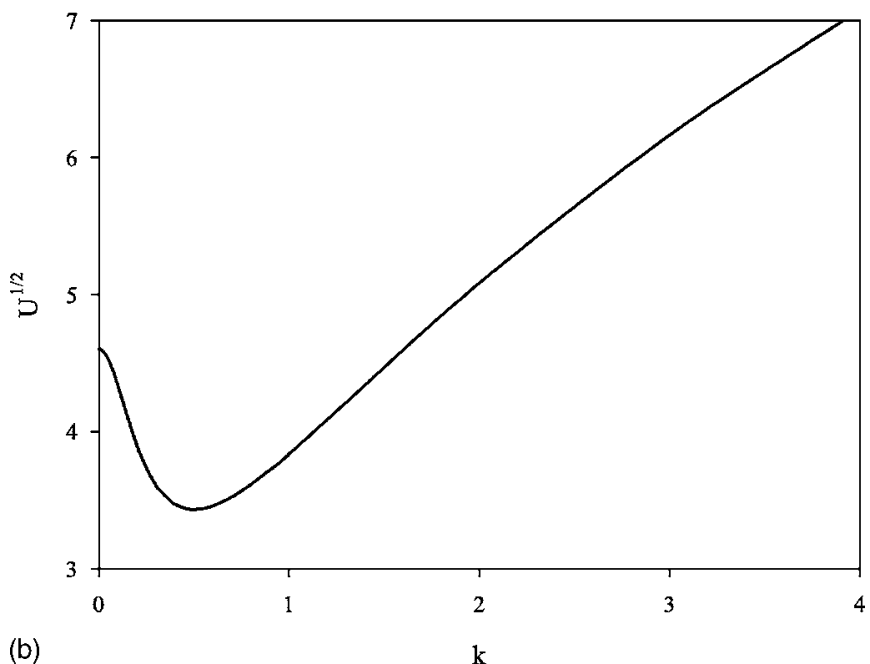

FIG. 2. Dispersion relation $U(k)\left[C=10, L=12.5, \mathrm{Bo}=0.347, g^{*}=0.047\right.$, $\epsilon_{r}=4.69, \rho_{a} / \rho=1.25 \times 10^{-3}, M=9.6 \times 10^{-4}$ (the last three parameters correspond to castor oil)]. (a) $S=1 \times 10^{-4}\left(Q_{s 0}>0\right)$; a comparison of results taking into account (solid line) and neglecting (dotted line) the liquid motion. (b) $S=5 \times 10^{-2}\left(Q_{s 0}<0\right)$.

which $Q_{s 0}>0$ and $Q_{s 0}<0$, respectively. $U(k)$ is the critical value of the stability parameter $U$ corresponding to marginal stability of normal modes with wavenumber $k$, for fixed values of the rest of parameters. Since an arbitrary perturbation will generally contain all possible modes, the minimum of the curve $U(k)$ gives the true critical value of the stability parameter $U$, above which the system is unstable against linear perturbations. As it can be seen in the pictures the critical value of $U$ is smaller for $Q_{s 0}>0$.

The dispersion diagram obtained by neglecting the velocity of the liquid in the computation has also been included in Fig. 2(a). In this curve $U$ always increases with $k$, so that the minimum value is reached when $k=0$. This is in sharp contrast with the dispersion diagram obtained when the liquid velocity is included in the calculation, where the minimum is always reached at a finite value of the wavenumber $k$. The liquid motion plays a stabilizing role for small wavenumber perturbations. The effect of liquid motion is less noticeable for $k$ greater than 1 .

The other relevant parameter besides $U$ that can be eas-

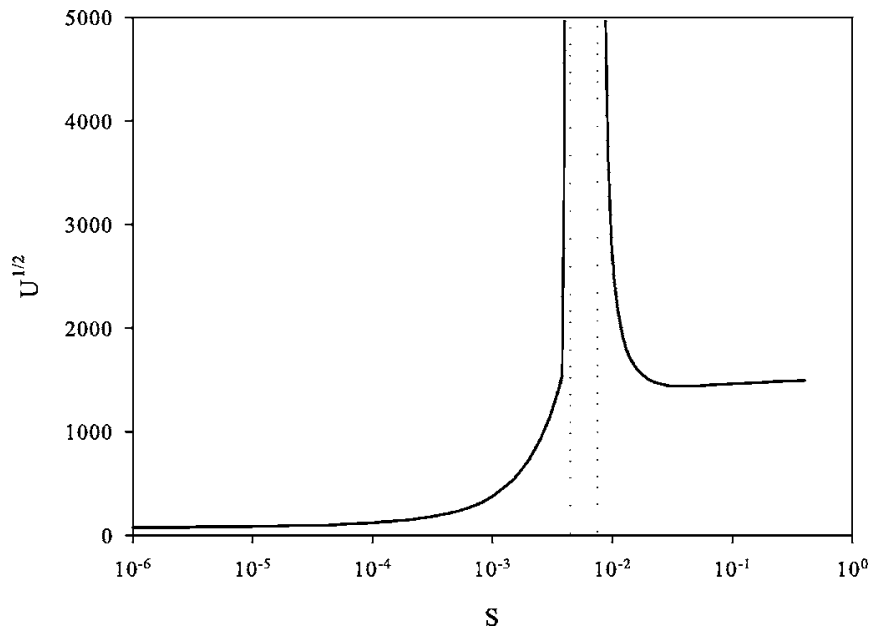

FIG. 3. The critical value of $U$ vs $S[C=10, L=12.5$, Bo $=0.446$, $g^{*}=1.029 \times 10^{4}, \epsilon_{r}=2.0, \rho_{a} / \rho=1.54 \times 10^{-3}, M=10.66 \times 10^{-4}$ (the last three parameters correspond to cyclohexane)]. The surface charge density $Q_{s 0}$ is positive on the left branch and negative on the right branch.

ily varied in an experiment, for a given liquid, is $S$. Figure 3 shows the dependence of the critical value $U$ upon $S$. Two branches appear in the plot, separated by a gap where no solution is found. Left and right branches correspond to values of $S$ for which $Q_{s 0}>0$ and $Q_{s 0}<0$, respectively. The gap limits roughly correspond to the values of $S$ that make zero the charge density and the electric pressure jump, respectively. The system is linearly stable in the gap for any value of the stability parameter $U$. It is important to realize that the existence of the gap does not mean that we can find an interval of values of conductivity of the liquid in which the static state remains stable, no matter how high the applied voltage $V$ is. In fact, in an actual experiment with a fixed value of the conductivity $\sigma$, when $V$ is turned on from zero $S$ takes very large values at the start, decreasing monotonically as $V$ is being raised. Thus, a value of $S$ will eventually be reached low enough for the system to become unstable.

A most interesting graph is obtained by drawing the stable and unstable regions in the $(\sigma, V)$ plane (Fig. 4). This graph is readily obtained from Fig. 3, by making the appropriate transformations between the experimentally measurable dimensional quantities $\sigma, V$ and the nondimensional parameters $S, U$. This diagram is susceptible of an experimental verification if the liquid conductivity is varied with the help of adequate additives. The values of the several liquid properties involved correspond to cyclohexane.

It is also of interest to study the variation of the critical voltage with the liquid layer thickness. The results obtained for castor oil, computed for values of the parameters for which experimental results were available, ${ }^{3}$ are shown in Fig. 5. We have plotted, for the purpose of comparing the critical voltage obtained, neglecting the liquid velocity. The experimental and theoretical values do not match exactly, but they follow the same trend: the critical voltage increases with the liquid layer thickness. The values computed taking into account the liquid motion are in better agreement with the experimental ones. The critical wavelength is nonzero when the velocity of the liquid is taken into account. The computed 


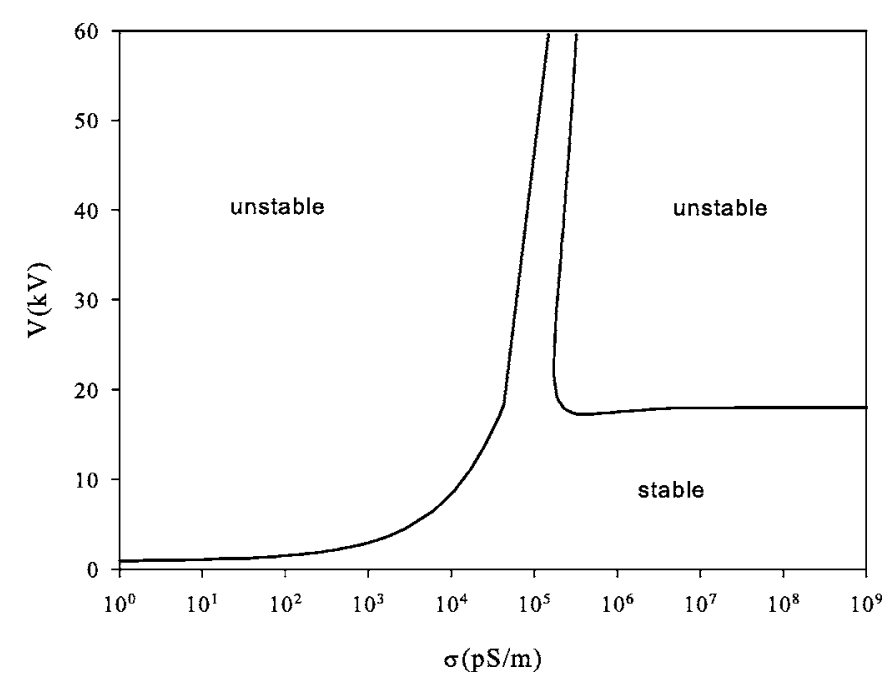

FIG. 4. Stability and instability regions for cyclohexane in the $(\sigma, V)$ plane $\left[\rho=779 \mathrm{~kg} / \mathrm{m}^{3}, \epsilon_{r}=2.0, \eta=10^{-3} \mathrm{~kg} /(\mathrm{m} \mathrm{s}), \gamma=0.0247 \mathrm{~N} / \mathrm{m}, \rho_{a}=1.2 \mathrm{~kg} / \mathrm{m}^{3}\right.$, $K=10^{-4} \mathrm{~m}^{2} /(\mathrm{V} \mathrm{s})$; injection level: $C=10$; geometric parameters: $d=1.2 \mathrm{~mm}, L=15 \mathrm{~mm}]$. The two oblique asymptotes are parallel in this semilogarithmic plot.

wavelength is of the order of $10 \mathrm{~mm}$, compatible with the observed typical size of the cells. Nevertheless, this comparison between theory and experiment must be considered with caution, and a more systematic comparison is desirable, for which new and more extensive measurements are needed.

\section{ANALYTICAL SOLUTION FOR $\boldsymbol{k} \rightarrow 0$}

The numerical algorithm described above breaks down when $k \rightarrow 0$. The reason is that for $k=0$, Eq. (78) reduces to $d^{2} u / d z^{2}(0)=0$, a boundary condition that cannot be satisfied together with Eqs. (73), (74), and (77). Fortunately, the ODEs can be somewhat simplified in this regime, thus allowing a closed form analytical solution.

The equation for the velocity of the liquid, Eq. (69), can be solved exactly for any value of $k$ :

$$
u(z)=\left(A_{1}+B_{1} z\right) e^{k z}+\left(C_{1}+D_{1} z\right) e^{-k z} .
$$

The constants are to be chosen so that the boundary conditions given by Eqs. (73), (74), and (77) are satisfied, which gives

$$
\begin{aligned}
& C_{1}=-A_{1}, \\
& B_{1}=-A_{1} \frac{2 k-1+e^{-2 k}}{2 k}, \\
& D_{1}=A_{1} \frac{2 k+1-e^{2 k}}{2 k} .
\end{aligned}
$$

The equation for the electric potential in the liquid, Eq. (68), can likewise be exactly solved:

$$
g(z)=A_{2} e^{k z}+B_{2} e^{-k z},
$$

where $B_{2}=-A_{2} e^{2 k}$ to satisfy the boundary condition given by Eq. (72).

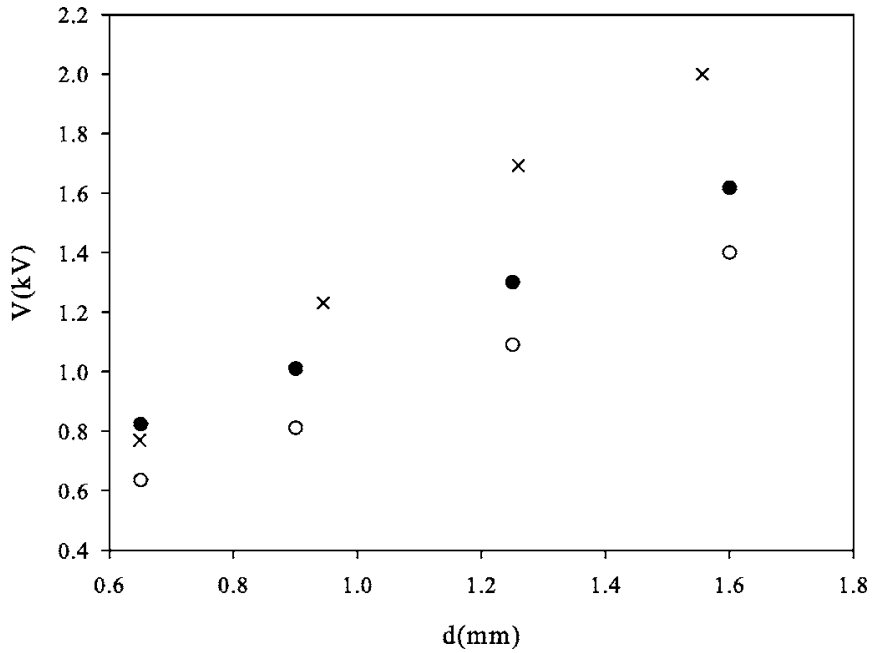

FIG. 5. Critical voltage as a function of liquid layer thickness for castor oil $\left[\rho=958 \mathrm{~kg} / \mathrm{m}^{3}, \quad \epsilon_{r}=4.69, \quad \sigma=7 \times 10^{-11} \mathrm{~s} / \mathrm{m}, \quad \eta=0.57 \mathrm{~kg} /(\mathrm{m} \mathrm{s}), \quad \gamma\right.$ $=0.039 \mathrm{~N} / \mathrm{m}, \rho_{a}=1.2 \mathrm{~kg} / \mathrm{m}^{3}, K=10^{-4} \mathrm{~m}^{2} /(\mathrm{V} \mathrm{s}) ;$ injection level: $C=10$; geometric parameters: $d=0.65,0.90,1.25$, and $1.60 \mathrm{~mm}, h=d+L=15 \mathrm{~mm}$ ]. A comparison between results, taking into account $(\bullet)$ and neglecting $(\bigcirc)$ the liquid motion. The experimental results $(\times)$ are from Ref. 3 .

No closed form solution can be found for the electric potential in the air, Eq. (67), when $k \neq 0$. However, for small values of $k$ that equation can be replaced to a certain degree of accuracy by the equation

$$
(b-L z)^{2} \frac{d^{3} h}{d z^{3}}-L(b-L z) \frac{d^{2} h}{d z^{2}}-\frac{L^{2}}{4} \frac{d h}{d z}=0,
$$

whose general solution is

$$
h(z)=A_{3}(b-L z)^{3 / 2}+B_{3}(b-L z)^{1 / 2}+C_{3},
$$

with the constants related by

$$
\begin{aligned}
& B_{3}=A_{3} 3(b-L), \\
& C_{3}=-A_{3} 4(b-L)^{3 / 2},
\end{aligned}
$$

in order to satisfy the boundary conditions given by Eqs. (70) and (71).

When the expressions obtained for $u(z), g(z)$, and $h(z)$ are introduced into the remaining boundary conditions at the interface, Eqs. (75)-(79), we obtain a homogeneous linear system of four equations in the unknowns $A_{1}, A_{2}, A_{3}$, and $f_{0}$ :

$$
\left(d_{i j}\right)\left(\begin{array}{c}
A_{1} \\
A_{2} \\
A_{3} \\
f_{0}
\end{array}\right)=0 .
$$

The matrix elements $d_{i j}$ are functions of the parameters $M, U, S, \mathrm{Bo}, g^{*}, L$, and $C$, as well as of the wavenumber $k$. The secular equation,

$$
\operatorname{det}\left(d_{i j}\right)=0,
$$

yields a dispersion relation that gives $U$ as a function of $k$, for given values of the parameters of the system.

Though it is possible to write down explicit general expressions for the matrix elements $d_{i j}$, they are too cumber- 
some to be substituted into the secular equation. We consider in the Appendices the different regimes of interest, solving the corresponding simplified secular equation in each case. The results are summarized in the following scheme (critical voltages are expressed as dimensional quantities).

- Perfect conductor $(S \rightarrow \infty)$ :

- Strong injection $(C \rightarrow \infty)$,

$$
V_{c}^{2}=\frac{4}{9} \frac{\rho}{\epsilon_{0}} g L^{3} \text {. }
$$

- Weak injection $(C \rightarrow 0)$,

$$
V_{c}^{2}=\frac{\rho}{\epsilon_{0}} g L^{3}
$$

- Insulating liquid $(S \rightarrow 0)$ :

- Strong injection $(C \rightarrow \infty)$,

$$
V_{c}^{2}=\rho \frac{d^{3}}{\epsilon} g \text {. }
$$

- Weak injection $(C \rightarrow 0)$,

$$
V_{c}^{2}=\frac{g\left(\rho-\rho_{a}\right)}{\epsilon} \frac{\left(d+\epsilon_{r} L\right)^{3}}{\left(1-\epsilon_{r}\right)^{2}} \text {. }
$$

The last two cases, of very insulating liquid, do not correspond to any physical situation, since at very low conductivities the liquids behave non-Ohmically. However, it is interesting to give these results as they may be compared with previous ones obtained by other authors.

The analytical solution has been very valuable to check the numerical code. We have verified that the numerical results, computed for values of $k$ as low as possible, coincide with the analytical solution for any choice of the parameters.

\section{CONCLUSION}

We have studied the linear stability of a horizontal liquid layer of finite conductivity subjected to charge injection from the air above. The critical values of the voltage we have computed are noticeably different from those obtained neglecting the liquid velocity. Also the shape of the dispersion relation $U(k)$ is different, having a minimum for $k \neq 0$ that is absent when velocity is not considered.

The variation of the critical voltage with the conductivity of the liquid exhibits two branches. The values of the critical voltage for low conductivities are smaller than those for higher conductivities, which makes the experimental observation of the instability easier for low conducting liquids.

We have calculated the critical voltage as a function of the liquid layer thickness for one particular liquid, castor oil. The dependence follows the same trend observed experimentally. The critical voltages obtained when the liquid velocity is taken into account in the computation are closer to experimental values, compared to those obtained neglecting liquid motion.

The problem has been solved analytically in the limit $k \rightarrow 0$. This solution allows to recover the most important limit situations (weak or strong injection, small or large conductivity). We have used this analytical solution to check exhaustively the accuracy of the numerical code.

\section{ACKNOWLEDGMENT}

This work has been carried out with financial support from the Spanish Ministerio de Ciencia y Tecnología (MCYT) under Research Project No. BFM2003-01739.

\section{APPENDIX A: STRONG INJECTION REGIME $(C \rightarrow \infty)$}

The constants $a$ and $b$ appearing in the nonperturbed solution are obtained from Eqs. (37) and (38). The substitution of $a$ from Eq. (38) into Eq. (37) leads to a single equation for $b$ :

$$
\frac{2 C^{2}}{S}(b-L)+\frac{4}{3} C(b-L)^{1 / 2}\left[b^{3 / 2}-(b-L)^{3 / 2}\right]=1 .
$$

For large values of $C$, the only possibility is $b=L+x$ with $x \ll L$. Thus, we get the approximate simplified equation:

$$
\frac{2 C^{2}}{S} x+\frac{4}{3} C x^{1 / 2} L^{3 / 2}=1,
$$

whose solution is

$$
x^{1 / 2}=\frac{L^{3 / 2}}{3 C} S\left[\left(1+\frac{9}{2 L^{3} S}\right)^{1 / 2}-1\right] \text {. }
$$

The value obtained for $x^{1 / 2}$ is an increasing function of $S$ varying from 0 when $S \rightarrow 0$, to $3 /\left(4 C L^{3 / 2}\right)$ when $S \rightarrow \infty$. Therefore, $x$ remains small in the whole range of values of $S$, consistently with the assumption made at the start.

The fact that $b \approx L$, besides the approximation of the exponentials for small values of $k$, greatly simplifies the expression of the interface boundary conditions, Eq. (89), which can now be written as

$$
\begin{gathered}
\left(\begin{array}{cccc}
0 & 2 k & b^{3 / 2} & c_{1} \\
\frac{2}{3} k^{3} M c_{2} b^{1 / 2} U^{-1 / 2} & -2 k S b & a b\left(\frac{3}{2}-k^{2} b^{2}\right) & 0 \\
\frac{8}{3} & 2 c_{3} U & 0 & \frac{1}{k} c_{3} \frac{a^{2}}{2 S} U \\
-4 & \epsilon_{r} \frac{a^{2}}{S} U & -\frac{1}{k} a b U & \frac{1}{k} c_{4}
\end{array}\right) \\
\times\left(\begin{array}{c}
A_{1} \\
A_{2} \\
A_{3} \\
f_{0}
\end{array}\right)=0, \\
\end{gathered}
$$

where $c_{1}, c_{2}, c_{3}$, and $c_{4}$ are defined as 


$$
\begin{aligned}
& c_{1}=\frac{a^{2}}{2 S}-a b^{1 / 2}, \quad c_{2}=c_{3} b^{1 / 2}, \quad c_{3}=\epsilon_{r} \frac{a^{2}}{2 S}-a b^{1 / 2}, \\
& c_{4}=1-\frac{\rho_{a}}{\rho}+\frac{k^{2}}{\mathrm{Bo}} .
\end{aligned}
$$

The secular equation, Eq. (90), finally takes the form

$$
p_{3} U^{3 / 2}+p_{2} U+p_{1} U^{1 / 2}+p_{0}=0,
$$

with the coefficients given by

$$
\begin{gathered}
p_{3}=\frac{1}{6} M \frac{c_{2} c_{3}}{c_{1}} a^{2} b\left(1+\epsilon_{r} \frac{a^{2}}{4 S^{2}}\right) k^{2}, \\
p_{2}=-\frac{c_{3}}{c_{1}} a^{2} b^{1 / 2}\left(\frac{b}{2}+\frac{3}{2}-k^{2} b^{2}\right)+\frac{2}{3} a\left[\epsilon_{r} \frac{a^{2}}{2 S}\left(\frac{3}{2}-k^{2} b^{2}\right)-S b\right], \\
p_{1}=-\frac{1}{6} M \frac{c_{2} c_{3} c_{4}}{c_{1}} b k^{2}, \\
p_{0}=-\frac{2}{3} \frac{c_{4}}{c_{1}}\left[a\left(\frac{3}{2}-k^{2} b^{2}\right)+S b^{3 / 2}\right] .
\end{gathered}
$$

The roots of Eq. (A6) can be obtained numerically, and the least positive root is to be compared with the result obtained in Sec. III from the numerical solution of the exact ordinary differential equations.

The solution corresponding to $S \rightarrow \infty$ and $k \rightarrow 0$, a problem studied in Ref. 17, can be obtained at once from Eq. (A6) by setting $k=0$ :

$$
\left(\begin{array}{cccc}
0 & 2 k & 3 L b^{1 / 2} & c_{1} \\
\frac{2}{3} k^{3} M c_{2} b^{1 / 2} U^{-1 / 2} & -2 k S b & a b\left(\frac{3}{2}-k^{2} 3 L b\right) & 0 \\
\frac{8}{3} & 2 c_{3} U & 0 & \frac{1}{k} c_{3} \frac{a^{2}}{2 S} U \\
-4 & \epsilon_{r} \frac{a^{2}}{S} U & -\frac{1}{k} 3 a b U & \frac{1}{k} c_{4}
\end{array}\right)\left(\begin{array}{c}
A_{1} \\
A_{2} \\
A_{3} \\
f_{0}
\end{array}\right)=0
$$

$$
\begin{aligned}
& p_{1}=-\frac{1}{2} M \frac{c_{2} c_{3} c_{4}}{c_{1}} L k^{2}, \\
& p_{0}=-\frac{2}{3} \frac{c_{4}}{c_{1}}\left[a\left(\frac{3}{2}-k^{2} 3 L b\right)+3 S b^{1 / 2} L\right] .
\end{aligned}
$$

$$
\begin{aligned}
\lim _{k \rightarrow 0} U(k) & =-\frac{p_{0}(k=0)}{p_{2}(k=0)} \\
& =\frac{\frac{2}{3} \frac{c_{4}}{c_{1}}\left(a \frac{3}{2}+S b^{3 / 2}\right)}{-\frac{c_{3}}{c_{1}} a^{2} b^{1 / 2}\left(\frac{b}{2}+\frac{3}{2}\right)+\frac{2}{3} a\left(\epsilon_{r} \frac{a^{2}}{2 S} \frac{3}{2}-S b\right)} .
\end{aligned}
$$

Now $a \approx 3 /\left(2 L^{3 / 2}\right)$ and $b \approx L$, so when $S \rightarrow \infty$ we get $U \rightarrow(4 / 9) g^{*} L^{3}$. This is the limit value obtained in Ref. 17 .

Similarly, the solution corresponding to $k \rightarrow 0$ and $S \rightarrow 0$ can be readily obtained from Eq. (A6), giving $U \rightarrow g^{*} / \epsilon_{r}$.

\section{APPENDIX B: WEAK INJECTION REGIME $(C \rightarrow 0)$}

\section{Liquid with high conductivity $(S \rightarrow \infty)$}

The stability of a horizontal interface between air and a highly conducting liquid under the influence of an initially uniform vertical electric field, when no charge injection is present, was thoroughly studied by Taylor and McEwan. ${ }^{8}$ The equation that gives the parameters of the unperturbed

$$
\frac{4}{3} C(b-L)^{1 / 2}\left[b^{3 / 2}-(b-L)^{3 / 2}\right]=1 .
$$

When $C \rightarrow 0$, it must be $b \rightarrow \infty$, so we get

$$
a \approx\left(\frac{2 C}{L}\right)^{1 / 2}, \quad b \approx \frac{1}{2 C L} .
$$

The fact that $b \gg L$ leads to the following expression for the interface boundary conditions, Eq. (89):
The secular equation takes the same form as Eq. (A6), with coefficients given now by

$$
\begin{aligned}
p_{3}= & \frac{1}{2} M \frac{c_{2} c_{3}}{c_{1}} a^{2} b\left(1+\epsilon_{r} \frac{a^{2}}{4 S^{2}} \frac{L}{b}\right) k^{2}, \\
p_{2}= & -\frac{c_{3}}{c_{1}} a^{2} b^{1 / 2} 3\left(\frac{L}{2}+\frac{1}{2}-k^{2} L b\right) \\
& +\frac{2}{3} a\left[\epsilon_{r} \frac{a^{2}}{2 S}\left(\frac{3}{2}-3 k^{2} L b\right)-3 S b\right],
\end{aligned}
$$




$$
\begin{aligned}
\lim _{k \rightarrow 0} U(k)= & -\frac{p_{0}(k=0)}{p_{2}(k=0)} \\
= & \frac{\frac{2}{3} \frac{c_{4}}{c_{1}}\left(a \frac{3}{2}+3 S b^{1 / 2} L\right)}{-\frac{c_{3}}{c_{1}} a^{2} b^{1 / 2} 3\left(\frac{L}{2}+\frac{1}{2}\right)+\frac{2}{3} a\left(\epsilon_{r} \frac{a^{2}}{2 S} \frac{3}{2}-3 S b\right)} .
\end{aligned}
$$

Taking into account that now $a \approx(2 C / L)^{1 / 2}$ and $b \approx 1 /(2 C L)$, when $S \rightarrow \infty$, we finally get $U \rightarrow g^{*} L^{3}$. This is the asymptotic value obtained in Ref. 8 .

\section{Insulating liquid $(S \rightarrow 0)$}

The last asymptotic regime that will be considered is that of an horizontal interface between two insulating fluids, again when no charge injection is present, which was studied by Melcher. ${ }^{10}$ Equation (A1) should now be carefully analyzed, since no unambiguous behavior can be ascertained for $b$ when $C \rightarrow 0$ and $S \rightarrow 0$ : different solutions could be obtained depending on how fast $C$ and $S$ separately approach zero. However, in the case considered by Melcher no surface charge density is supported by the interface. Therefore, we must let $C \rightarrow 0$ and $S \rightarrow 0$ but subjected to the constraint

$$
Q_{s 0}=\epsilon_{r} \frac{a^{2}}{2 S}-a b^{1 / 2}=0
$$

Thus, Eq. (A1) takes now the form

$$
2 C(b-L)^{1 / 2}\left[\frac{1}{\epsilon_{r}} b^{1 / 2}+\frac{2}{3}\left(b^{3 / 2}-(b-L)^{3 / 2}\right)\right]=1 .
$$

When $C \rightarrow 0$ it should be $b \rightarrow \infty$, and we arrive at the solution

$$
a \approx\left(\frac{2 C}{\frac{1}{\epsilon_{r}}+L}\right)^{1 / 2}, \quad b \approx \frac{1}{2 C\left(\frac{1}{\epsilon_{r}}+L\right)} .
$$

Since $b \rightarrow \infty$, the interface boundary conditions lead to the same system of equations obtained in the previous section, Eq. (B3). Likewise Eq. (B8) holds in this case. But now $c_{3}=0$ and $c_{1}=\left(1 / \epsilon_{r}-1\right) a b^{1 / 2}$, which yields

$$
\lim _{k \rightarrow 0} U(k)=\frac{c_{4}\left(\frac{1}{\epsilon_{r}}+L\right)^{2}\left(\frac{1}{2} a+S b^{1 / 2} L\right)}{\left(\frac{1}{\epsilon_{r}}-1\right)\left(\frac{1}{2} a-S b^{1 / 2}\right)} .
$$

Multiplying the numerator and denominator by $a$ and taking into account that $a^{2} / 2=S a b^{1 / 2} / \epsilon_{r}$ directly gives

$$
\lim _{k \rightarrow 0} U(k)=g^{*}\left(1-\frac{\rho_{a}}{\rho}\right) \frac{\left(\frac{1}{\epsilon_{r}}+L\right)^{3}}{\left(\frac{1}{\epsilon_{r}}-1\right)^{2}},
$$

which is the result obtained in Ref. 10 .

${ }^{1}$ A. T. Pérez, "EHD instabilities induced by corona discharge," Proceedings of 12th International Conference on Dielectric Liquids (ICDL '96), Roma, Italy (IEEE Press, Piscataway, NJ, 1996), pp. 126-129.

${ }^{2}$ A. T. Pérez, "Rose-window instability in low conducting liquids," J. Electrost. 40-41, 141 (1997).

${ }^{3}$ F. Vega and A. T. Pérez, "Corona-induced electrohydrodynamic instabilities in low conducting liquids," Exp. Fluids 34, 726 (2003).

${ }^{4}$ C. S. Herrick, "Electroconvection cells in dielectric liquids interfaced with conducting fluids," Proc. R. Soc. London, Ser. A 336, 487 (1974).

${ }^{5} \mathrm{P}$. Atten and R. Moreau, "Stabilité électrohydrodynamique des liquides isolants soumis à une injection unipolaire,” J. Mec. 11, 471 (1972).

${ }^{6}$ P. K. Watson, J. M. Schneider, and H. R. Till, "Electrohydrodynamic stability of space-charge-limited currents in dielectric liquids. II," Phys. Fluids 13, 1955 (1970).

${ }^{7}$ A. Castellanos, Electrohydrodynamics (Springer-Verlag, Vienna, 1998).

${ }^{8}$ G. I. Taylor and A. D. McEwan, "The stability of a horizontal fluid interface in a vertical electric field," J. Fluid Mech. 22, 1 (1965).

${ }^{9}$ J. R. Melcher and C. V. Smith Jr., "Electrohydrodynamic charge relaxation and interfacial perpendicular-field instability," Phys. Fluids 12, 778 (1969).

${ }^{10}$ J. R. Melcher, Field-Coupled Surface Waves (MIT Press, Cambridge, 1963).

${ }^{11}$ F. Vega and A. T. Pérez, "Instability in a non-Ohmic/Ohmic fluid interface under a perpendicular electric field and unipolar injection," Phys. Fluids 14, 2738 (2002).

${ }^{12}$ J. R. Melcher and G. I. Taylor, "Electrohydrodynamics: a review of the role of interfacial shear stresses," Annu. Rev. Fluid Mech. 1, 111 (1969).

${ }^{13}$ A. Castellanos and A. González, "Non-linear electrohydrodynamics of free surfaces,” IEEE Trans. Dielectr. Electr. Insul. 5, 334 (1998).

${ }^{14} \mathrm{~J}$. Lighthill, Waves in Fluids (Cambridge University Press, Cambridge, 2002).

${ }^{15} \mathrm{~S}$. Chandrasekhar, Hydrodynamic and Hydromagnetic Stability (Dover, New York, 1981).

${ }^{16}$ J. Kierzenka and L. F. Shampine, "A BVP solver based on residual control and the Matlab PSE," ACM Trans. Math. Softw. 27, 299 (2001).

${ }^{17}$ P. Atten and D. Koulova-Nenova, "On the instability between two layers of conducting and insulating liquids subjected to a DC field," Proceedings of 13th International Conference on Dielectric Liquids (ICDL '99), Nara, Japan (IEEE Press, Piscataway, NJ, 1999), pp. 277-280. 